\title{
Influence of mutual drag of light and heavy holes on magnetoresistivity and Hall-effect of $p$-silicon and $p$-germanium
}

\author{
I.I. Boiko \\ V. Lashkaryov Institute of Semiconductor Physics, NAS of Ukraine, \\ 45, prospect Nauky, 03028 Kyiv, Ukraine \\ E-mail: igorboiko@yandex.ru; phone:+38(044)236-5422
}

\begin{abstract}
Hall-effect and magnetoresistivity of holes in silicon and germanium are considered with due regard for mutual drag of light and heavy band carriers. Search of contribution of this drag shows that this interaction has a sufficient influence on both effects.
\end{abstract}

Keywords: quantum kinetic equation, Hall-effect, magnetoresistivity, interband drag.

Manuscript received 29.03.11; revised manuscript received 29.08.11; accepted for publication 14.09.11; published online 30.11.11.

\section{Introduction}

In the previous work (see Ref. [1]), we investigated the influence of mutual drag of heavy and light holes on conductivity of $p$-germanium and $p$-silicon. It was shown that this drag significantly diminishes the total conductivity of holes. In practice, this effect attracted attention of a great number of earlier investigators. One of the reasons was vain attempts to describe mutual drag of band carriers belonging to different groups (see, for example, Refs. [2, 3]) by using the wide-spread tauapproximation in the course of solution of kinetic equation (see Refs. [4, 5]). In this work, we have used the method of balance equation (see Refs. [6-8]), which allows to introduce into consideration mutual drag of carriers from two bands that close up in the center of wavevector space. For simplicity of calculations, we accept here spherical bands approximation. So, the dispersion law for holes has the following simple form:

$\varepsilon_{\vec{k}}^{(a)}=\hbar^{2} k^{2} / 2 m_{a} \quad(a=1$ or 2$)$.

In this formula, $m_{a}$ is the effective masses ( $m_{1}$ is mass of light holes, and $m_{2}$ is mass of heavy holes).

\section{Balance equations}

Let us consider the set of two balance equations obtained as a first momentum of quantum kinetic equations (see Refs. [7, 9]):
$e\left[\vec{E}+(1 / c)\left(\vec{H} \times \vec{u}^{(a)}\right)\right]+\vec{F}^{(a)}+\sum_{b=1}^{2} \vec{F}^{(a, b)}=0(a=1,2)$.

Here vectors $\vec{E}$ and $\vec{H}$ represent electrical and magnetic fields, the values $\vec{u}^{(a)}$ are drift velocities of light and heavy holes, $\vec{F}^{(a)}$ is the resistant force related to an external scattering system, $\vec{F}^{(a, b)}$ is the force related with Coulomb interaction of heavy and light holes.

We restrict here our consideration by external scattering system containing charged impurities and acoustic phonons.

Accepting the model of non-equilibrium distribution functions of holes from different groups as Fermi functions with argument containing for $a$-group the shift of velocity $\vec{v}^{(a)}(\vec{k})=\hbar^{-1}\left(\partial \varepsilon_{\vec{k}}^{(a)} / \partial \vec{k}\right)$ on correspondent velocity $\vec{u}^{(a)}$

$f_{\vec{k}}^{(a)}=f^{0(a)}\left(\vec{v}^{(a)}(\vec{k})-\vec{u}^{(a)}\right) \quad(a=1,2)$

(here $f^{0(a)}\left(\vec{v}^{(a)}(\vec{k})\right)$ is equilibrium distribution function for $a$-carriers) we obtain the following expressions for forces presented in Eq. (2): $\vec{F}^{(a)}=-e \beta^{(a)} \vec{u}^{(a)} ; \vec{F}^{(a, b)}=-e \xi^{(a, b)}\left(\vec{u}^{(a)}-\vec{u}^{(b)}\right)$. 

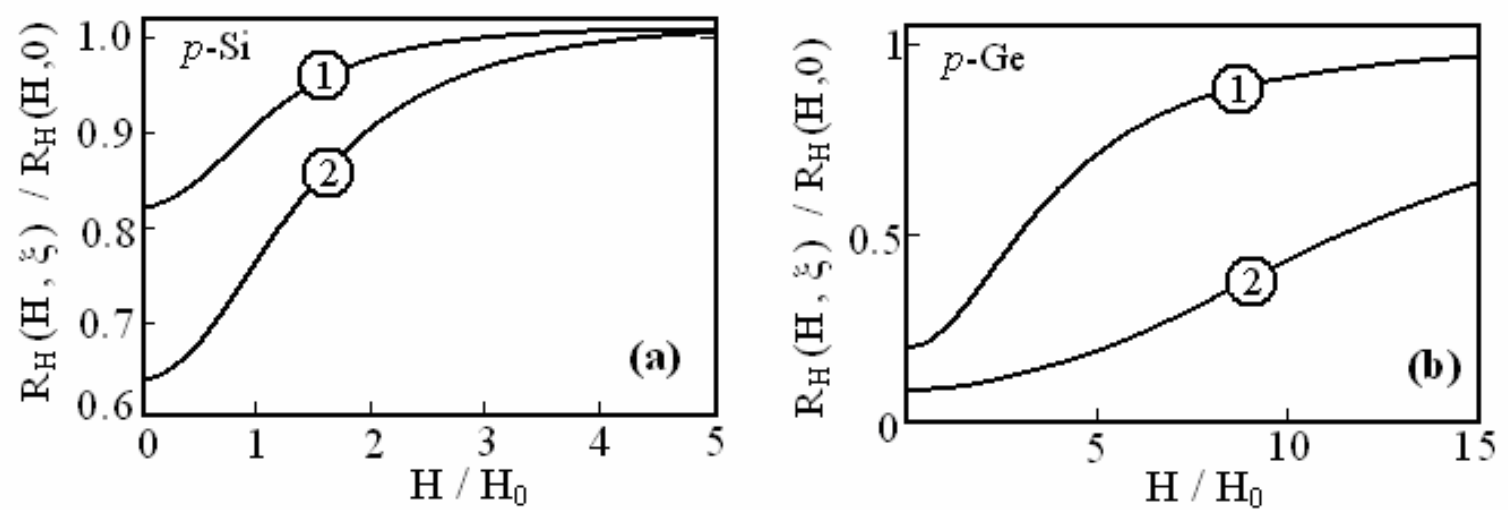

Fig. 1. Influence of interband drag on the Hall coefficients. (a) $p$-silicon: $p=10^{17} \mathrm{~cm}^{-3}$; (b) $p$-germanium: $p=10^{14} \mathrm{~cm}^{-3}$. $1-T=50 \mathrm{~K}, 2-100 \mathrm{~K}$.
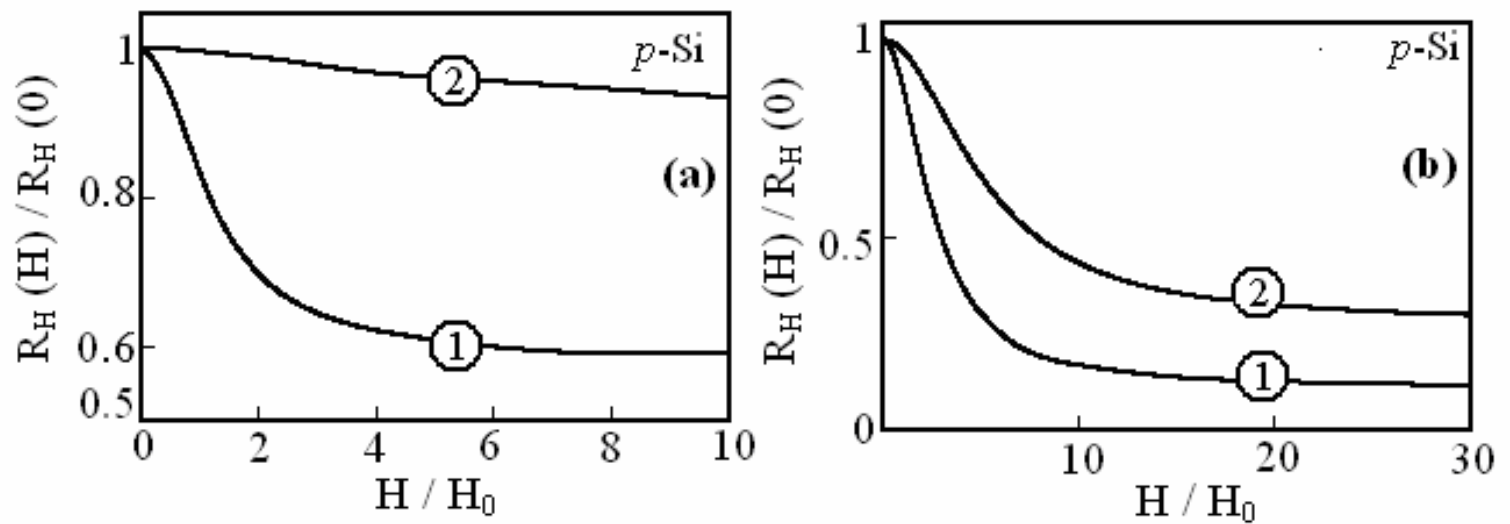

Fig. 2. Dependence of the relative Hall coefficient of $p$-silicon on the dimensionless magnetic field. $p=10^{14} \mathrm{~cm}^{-3}$. (a) $T=50 \mathrm{~K}$, (b) $100 \mathrm{~K} .1-\xi=0,2-\xi \neq 0$.

In what follows, we shall consider only nondegenerate band carriers. Then (see Ref. [10])

$\beta^{(a)}=\lambda^{(a)}+\chi^{(a)}$

$\lambda^{(a)}=\frac{4 \sqrt{2 \pi m_{a}} e^{3} n_{I}}{3\left(k_{B} T\right)^{3 / 2} \varepsilon_{L}^{2}} \int_{0}^{\infty} \frac{q^{3} d q}{\left(q^{2}+q_{0}^{2}\right)^{2}} \exp \left(-\frac{\hbar^{2} q^{2}}{8 m_{a} k_{B} T}\right) ;$

$\chi^{(a)}=\frac{8 \sqrt{2} \Xi_{A}^{2}\left(k_{B} T\right)^{3 / 2} m_{a}^{5 / 2}}{3 \pi^{3 / 2} \hbar^{4} e \rho s^{2}} ;$

$\xi^{(a, b)}=\frac{8 \gamma e^{3} m_{b}^{2} p_{a}}{3 k_{B} T \hbar m_{a}} \times$

$\times \int_{0}^{\infty} \frac{q^{2} d q}{\left(q^{2}+q_{0}^{2}\right)^{2}} \exp \left[-\frac{\hbar^{2} q^{2}}{8 k_{B} T}\left(\frac{1}{m_{1}}+\frac{1}{m_{2}}\right)\right]$;

$\gamma=\int_{-\infty}^{\infty} \frac{w^{2} d w}{\sinh ^{2} w} \approx 3.29$

$q_{0}^{2}=\frac{4 \pi e^{2}\left(p_{1}+p_{2}\right)}{\varepsilon_{L} k_{B} T}$.
Here, $p_{a}$ is the density of $a$-holes, $n_{I}$ is the density of charged impurities. We assume $n_{I}=p=p_{1}+p_{2}$.

For nondegenerate carriers

$\frac{p_{1}}{p_{2}}=w=\left(\frac{m_{1}}{m_{2}}\right)^{3 / 2}$.

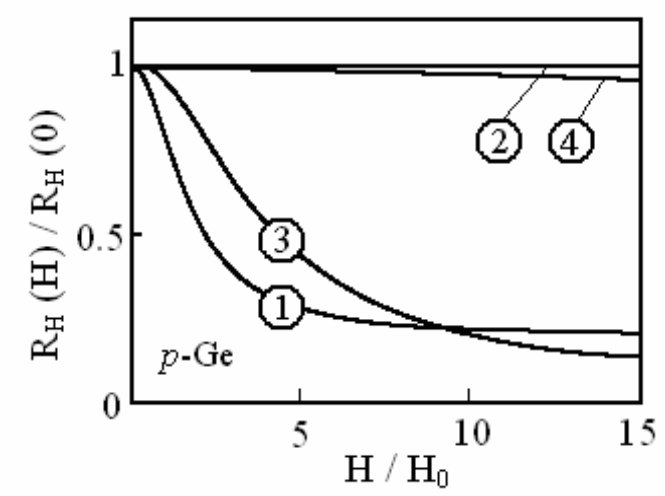

Fig. 3. Dependence of the relative Hall coefficient of $p$ germanium on the dimensionless magnetic field. $p=10^{14} \mathrm{~cm}^{-3}$. $T=50 \mathrm{~K}: 1-\xi=0,2-\xi \neq 0 . T=100 \mathrm{~K}: 3-\xi=0,4-\xi \neq 0$.

(C) 2011, V. Lashkaryov Institute of Semiconductor Physics, National Academy of Sciences of Ukraine 

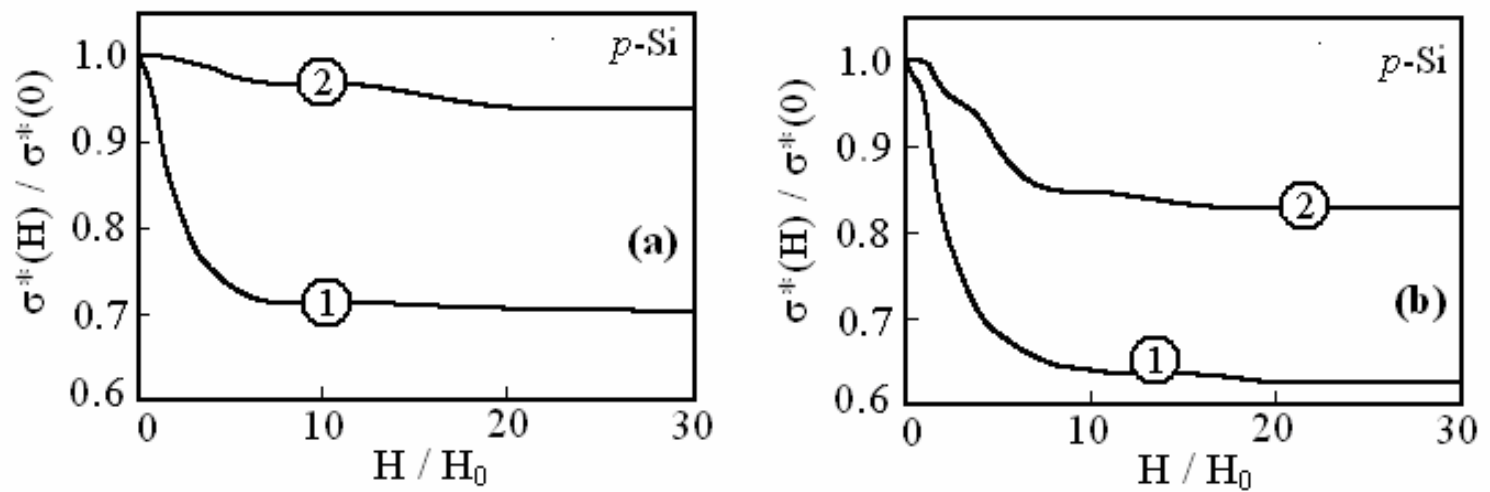

Fig. 4. Dependence of the relative magnetoconductivity of $p$-silicon on the dimensionless magnetic field. (a) $T=50 \mathrm{~K}$; (b) $T=$ 100 K. $1-\xi=0,2-\xi \neq 0 ; p=10^{14} \mathrm{~cm}^{-3}$.
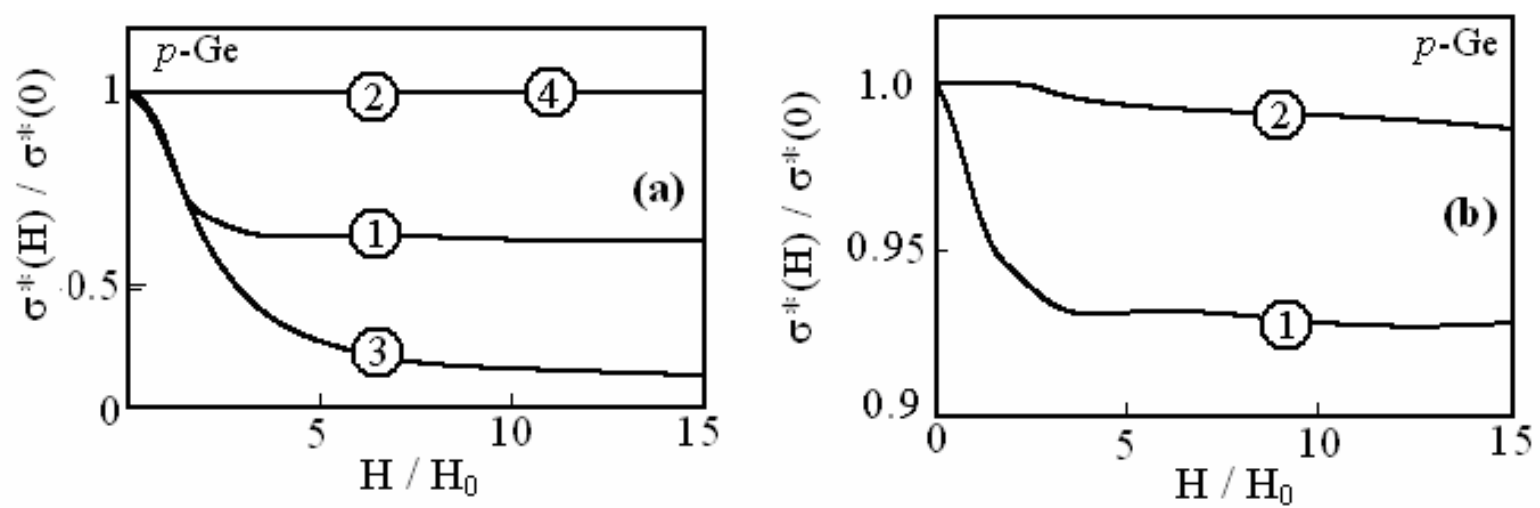

Fig. 5. Dependence of the relative magnetoconductivity of $p$-germanium on the dimensionless magnetic field. (a) $p=10^{14} \mathrm{~cm}^{-3}$; $T=50 \mathrm{~K}: 1-\xi=0,2-\xi \neq 0 . T=100 \mathrm{~K}: 3-\xi=0,4-\xi \neq 0$. (b) $p=10^{17} \mathrm{~cm}^{-3} ; T=100 \mathrm{~K}: 1-\xi=0,2-\xi \neq 0$.

For $p$-germanium $w=0.042$, for $p$-silicon $w=0.153$ (see Ref. [5]).

From the formulae (2) and (4), one obtains the system of equations for drift velocities:

$\vec{E}+(1 / c)\left(\vec{H} \times \vec{u}^{(a)}\right)-\beta^{(a)} \vec{u}^{(a)}-$

$-\sum_{b=1}^{2} \xi^{(a, b)}\left(\vec{u}^{(a)}-\vec{u}^{(b)}\right)=0 .(a=1,2)$.

The total density of current is

$\vec{j}=\sum_{a=1}^{2} \vec{j}^{(a)}=e \sum_{a=1}^{2} p_{a} \vec{u}^{(a)}$.

\section{Hall-coefficient and magnetoresistivity}

Let us consider the case when the external magnetic field $\vec{H}$ is directed along $z$-axis and total current along $x$-axis:

$\vec{H}=\left(0,0, H_{z}\right) \quad \vec{j}=\left(j_{x}, 0,0\right)$.

Then the electrical field $\vec{E}$ has the following components:

$\vec{E}=\left(E_{x}, E_{y}, 0\right)$.
Here the component $E_{x}$ is related to applied electrical field.

The relation between measured components of the total current and electrical field can be represented in the form

$j_{x}=\sigma^{*}(H) E_{x}$.

The scalar value $\sigma^{*}(H)$ is called by us as magnetoresistivity.

Let us introduce the Hall coefficient $R_{H}$ by using the following relation:

$R_{H}=\left|\frac{E_{y}}{H_{z} j_{x}}\right|$.

To calculate the values $\sigma^{*}(H)$ and $R_{H}$, the system of equations (12) should be solved at the first stage. For the case (14) and (15), we have the system of five equations:

$\vec{E}+(1 / c)\left(\vec{H} \times \vec{u}^{(1)}\right)=\beta^{(1)} \vec{u}^{(1)}+\xi\left(\vec{u}^{(1)}-\vec{u}^{(2)}\right) ;$

$\vec{E}+(1 / c)\left(\vec{H} \times \vec{u}^{(2)}\right)=$

$=\beta^{(2)} \vec{u}^{(2)}+\xi w^{-1}\left(\vec{u}^{(2)}-\vec{u}^{(1)}\right)$;

$p_{1} u_{y}^{(1)}+p_{2} u_{y}^{(2)}=0$ 
Here, $\quad \xi=\xi^{(1,2)} \quad$ (see Eqs. (8) and (11)); $\vec{u}^{(a)}=\left(u_{x}^{(a)}, u_{y}^{(a)}, 0\right)(a=1,2)$. The unknown values are $u_{x}^{(1)}, u_{y}^{(1)}, u_{x}^{(2)}, u_{y}^{(2)}$ and $E_{y}$.

Analytical solution of the system (18) is very simple but rather complicated in the form. Therefore we present here the results of our numerical calculations only by figures (here $H_{0}=c \beta^{(1)}$ ). To carry out the calculations, we used the following numerical values: $\varepsilon_{L}=12, \rho s^{2}=1.66 \cdot 10^{11} \mathrm{~Pa}, \Xi_{A}=-4.2 \mathrm{eV} \quad$ for $p$ silicon and

$\varepsilon_{L}=16, \rho s^{2}=1.26 \cdot 10^{11} \mathrm{~Pa}, \Xi_{A}=1.9 \mathrm{eV} \quad$ for $\quad p$ germanium.

\section{Results of calculations}

Fig. 1 gives the possibility to compare Hall coefficients calculated for the case when interband drag is taken into account $\left(R_{H}(H, \xi)\right)$ and is not taken $\left(R_{H}(H, 0)\right)$. One can see that $R_{H}(H, \xi)<R_{H}(H, 0)$, and the difference is especially significant at small magnetic field.

Figs. 2 and 3 show dependence of the Hall coefficient on the intensity of magnetic field. It follows that drag makes this dependence more smooth (in some cases this dependence practically disappears; see Fig. 3, the curve 2).

Figs. 4 and 5 represent the dependence of relative magnetoconductivity of $p$-silicon and $p$-germanium on dimensionless magnetic field. Calculations show that the influence of intensity of the field on conductivity is significantly moderated by interband drag (especially in germanium where the difference between effective masses of light and heavy holes is aparently high).

\section{References}

1. I.I. Boiko, Electron-electron drag in crystals with many-valley band // Semiconductor Physics, Quantum Electronics \& Optoelectronics 12 (3), p. 212-217 (2009).

2. J. Appel, A.W. Overhauser, Cyclotron resonance in two interacting electron systems with application to Si inversion layers // Phys. Rev. B, 18, p. 758 (1978).

3. C.A. Kukkonnen, P.M. Maldague, Electron-hole scattering and the electrical resistivity of the semimetal $\mathrm{TiS}_{2} / /$ Phys. Rev. Lett. 37, p. 782 (1976).

4. C. Herring, E. Vogt, Transport and deformationpotential theory for many-valley semiconductors with anisotropic scattering // Phys. Rev. 101, p. 944 (1956).

5. A.I. Anselm, Introduction to the Theory of Semiconductors. Nauka, Moscow, 1978 (in Russian).

6. P.N. Argyres, Force-balance theory of resistivity // Phys. Rev. B, 39, p. 2982 (1989).

7. I.I. Boiko, Transport of Carriers in Semiconductors. Publ. V. Lashkaryov Institute of Semiconductor Physics, NAS of Ukraine, Kyiv, 2009 (in Russian).

8. I.I. Boiko, Kinetics of Electron Gas Interacting with Fluctuating Potential. Naukova dumka, Kiev, 1993 (in Russian).

9. I.I. Boiko, Electron-electron drag in crystals with a multivalley band. Magnetoresistivity and Hall-effect // Semiconductor Physics, Quantum Electronics \& Optoelectronics, 12 (4), p. 349-356 (2009).

10. I.I. Boiko, Influence of mutual drag of light and heavy holes on conductivity of $p$-silicon and $p$ germanium // Semiconductor Physics, Quantum Electronics \& Optoelectronics, 14 (3), p. 357-361 (2011). 